\title{
Loss of Smell in COVID-19 Patients: New Biomarkers
}

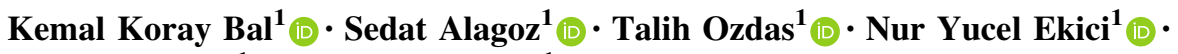 \\ Gokhan Kuran ${ }^{1}$ (1) $\cdot$ Orhan Görgülü̈ ${ }^{1}$ (B)
}

Received: 23 May 2021 / Accepted: 26 September 2021/Published online: 7 October 2021

(C) Association of Otolaryngologists of India 2021

\begin{abstract}
Patients with emerging anosmia may be asymptomatic carriers of coronavirus disease 2019 infection requiring self-isolation; otherwise, there are risks of facilitating the spread of the disease. This study aims to evaluate the loss of smell with visual analogue scale and to determine the relationship between the loss of smell and blood parameters.All patients' coronavirus disease 2019 swab cultures were polymerase chain reaction positive and pneumonia was found in computed tomographies consistent with oronavirus disease 2019. The study was conducted on 114 patients hospitalized between 01.11.2020 and 31.12.2020 in the Otorhinolaryngology coronavirus disease 2019 Service of University of Health Sciences Adana City Training and Research Hospital and followed up by us.A score of 10 indicates that the olfactory function is completely normal in all patients undergoing visual analogue scale, and a score of 0 indicates that they cannot smell anything. Patients who received visual analogue scale scored 10 points were categorized as Group 1 and others as Group 2. Statistical significance level was determined as $p<0.05$. A statistically significant difference was found between Group 1 and Group 2 in terms of visual analogue scale smell score, neutrophil, lymphocyte, neutrophil-lymphocyte ratio. Visual analogue scale smell score, neutrophil count, neutrophil-lymphocyte ratio value were found to be lower in Group 2 and lymphocyte count was found to be higher. Neutrophil, lymphocyte,
\end{abstract}

Kemal Koray Bal

dr.kemalkoraybal@gmail.com

1 Otorhinolaryngology Department, University of Health Sciences Adana City Training and Research Hospital, Kışla Mahallesi, Dr. Mithat Özsan Bulvarı, 4522. Sokak No:1, Yüreğir, Adana, Turkey neutrophil/lymphocyte ratio, platelet, platelet/lymphocyte ratio, which are also used in nasal diseases, may be used to detect loss of smell, predict, and even determine the prognosis of loss of smell if supported by further studies.

Keywords COVID-19 - Olfaction disorders . Hematologic tests · Biomarkers · Prognosis

\section{Introduction}

Numerous articles have been published on the occurrence of anosmia in people infected with the novel coronavirus. Loss of smell rates of coronavirus disease 2019 (COVID19) positive patients from various countries of the world have been reported to vary between 34 and 68\% [1-4]. Otorhinolaryngologists have warned authorities that loss of smell is a strong COVID-19 symptom [5]. Correlation between emerging chemosensory dysfunction (loss of smell and taste) and COVID-19 positivity has been reported in Iran and Italy since the beginning of the COVID-19 outbreak. Loss of smell or taste may be considered a potential early symptom [3]. Patients with emerging anosmia may be asymptomatic carriers of COVID-19 infection requiring self-isolation; otherwise, there are risks of facilitating the spread of the disease [4]. Pathological studies in macaques infected with SARSCoV-2 have identified the expression of SARS-CoV-2 antigens in ciliary epithelial cells [6]. Damage to nerve damage during the proliferation of SARS-CoV-2 may explain the early stage of COVID-19 [7].

Visual analog scale (VAS) can be used for smell and taste assessment in COVID-19 patients. This is a fast, easy, and safe application to be used in daily practice. Other tests such as face-to-face olfactometry are not recommended 
because of the high risk of transmission to healthcare professionals [8]. Individual tests for smell identification such as The University of Pennsylvania Smell Identification Test (UPSIT) can be considered safe, but they need to be properly validated and applied in different country populations because they are culturally dependent [9].

This study aims to evaluate the loss of smell with VAS and to determine the relationship between the loss of smell and blood parameters.

\section{Materials and Methods}

The study was conducted on 114 patients hospitalized between 01.11.2020 and 31.12.2020 in the Otorhinolaryngology COVID-19 Service of University of Health Sciences Adana City Training and Research Hospital and followed up by us. All patients' COVID-19 swab cultures were PCR positive and pneumonia was found in computed tomographies (CT) consistent with COVID-19. Patients received appropriate medical treatments related to COVID19. Nasal and paranasal diseases affecting the olfactory function of all patients participating in the study were excluded. Paranasal sinus CT was performed in all patients after discharge, those with additional nasal and sinonasal pathologies were excluded from the study. Patients with loss of taste and/or smell before, patients who underwent otolaryngological surgery, patients who smoked and/or smoked tobacco products were excluded from the study. All patients were adults and over 18 years of age. Blood parameters, additional diseases, gender, age, and VAS results of the patients taken during hospitalization were recorded. A score of 10 indicates that the olfactory function is completely normal in all patients undergoing VAS, and a score of 0 indicates that they cannot smell anything. Patients who received VAS scored 10 points were categorized as Group 1 and others as Group 2. Neutrophil (NEU), lymphocyte (LYMP), platelet (PLT), neutrophil/lymphocyte ratio (NLR), platelet/lymphocyte ratio (PLR) were recorded among the blood parameters. It was confirmed that all patients participating in the study did not have a loss of smell before. Additional diseases are categorized as diabetes mellitus (DM), hypertension (HT), coronary artery disease (CAD), chronic obstructive pulmonary disease (COPD), asthma (AS), chronic renal failure (CRF), and malignancy (MLG).

Normal distribution control of continuous variables was evaluated with Shapiro-Wilk statistics in statistical analyses. Mann-Whitney $U$ test was used to compare two independent groups that did not fit the normal distribution. A Chi-square test was used for the categorical variables. Mean \pm standard deviation values were given for numerical variables whereas frequencies and percentages were used for categorical variables in summary statistics. Statistical significance level was determined as $\mathrm{p}<0.05$. All analyses were performed with IBM SPSS 22 package software trial version.

Permission was obtained from the Ministry of Health of Turkey for our study and approval was obtained from University of Health Sciences Adana City Training and Research Hospital Clinical Research Ethics Committee. (Meeting Number: 74, Decision Number: 1232, Date: 13.01.2021, Ministry of Health of Turkey Permit No: 2021-01-03T16_03_16).

\section{Results}

There were 114 patients in the study, 75 of them were male $(65.8 \%)$ and 39 were female $(34.2 \%)$. The ages of the patients vary between 30 and 80 years and the mean age is 58.3 years.

An additional disease was observed in 60 patients $(52.6 \%)$. The most common additional disease is HT with 24 patients. CAD, COPD, and $\mathrm{AS}$ are observed in 9 patients and DM, CRF, and MLG in 3 patients.

There were 63 patients $(55.3 \%)$ in Group 1 and 51 patients (44.7\%) in Group 2. Group 1 included 45 men and 18 women. Group 2 included 30 men and 21 women.

The mean VAS smell score was 8.04 in males and 7.31 in females. In Group 2, the mean score was 5.06. The mean VAS smell score was 5.1 in males and 5 in females in Group 2 (Figs. 1, 2).

The mean count of NEU of all patients was 6710 and the values ranged from 1600 to 16,000. The mean count of NEU in men was $6692.00 \pm 3312.25$ whereas that of women was $6746.15 \pm 3444.59$. The mean count of NEU in Group 1 was $7366.67 \pm 3581.72$ whereas it was $5900.00 \pm 2852.37$ in Group 2.

The mean count of LYMP of all patients was 1194 and the values ranged from 300 to 10,000 . The mean count of LYMP in men was $1288.00 \pm 1831.77$ whereas that of women was $1015.38 \pm 621.77$. The mean count of LYMP in Group 1 was $852.38 \pm 371.51$ whereas it was $1617.65 \pm 2190.13$ in Group 2 .

The mean count of PLT of all patients was 228,710 and the values ranged from 100,000 to 891,000 . The mean count of PLT in men was 201,320.00 $\pm 67,755.35$ whereas that of women was $281,384.62 \pm 189,903.66$. The mean count of PLT in Group 1 was 218,523.81 $\pm 77,057.34$ whereas it was $241,294.12 \pm 172,733.59$ in Group 2 .

The mean value of NLR of all patients was 8.61 and the values ranged from 0.76 to 26.6. The mean value of NLR in men was $8.76 \pm 6.72$ whereas that of women was $8.33 \pm 5.23$. The mean value of NLR in Group 1 was $10.49 \pm 6.54$ whereas it was $6.30 \pm 4.99$ in Group 2 . 
Fig. 1 Male VAS point in Group 2

Fig. 2 Female VAS point In Group 2
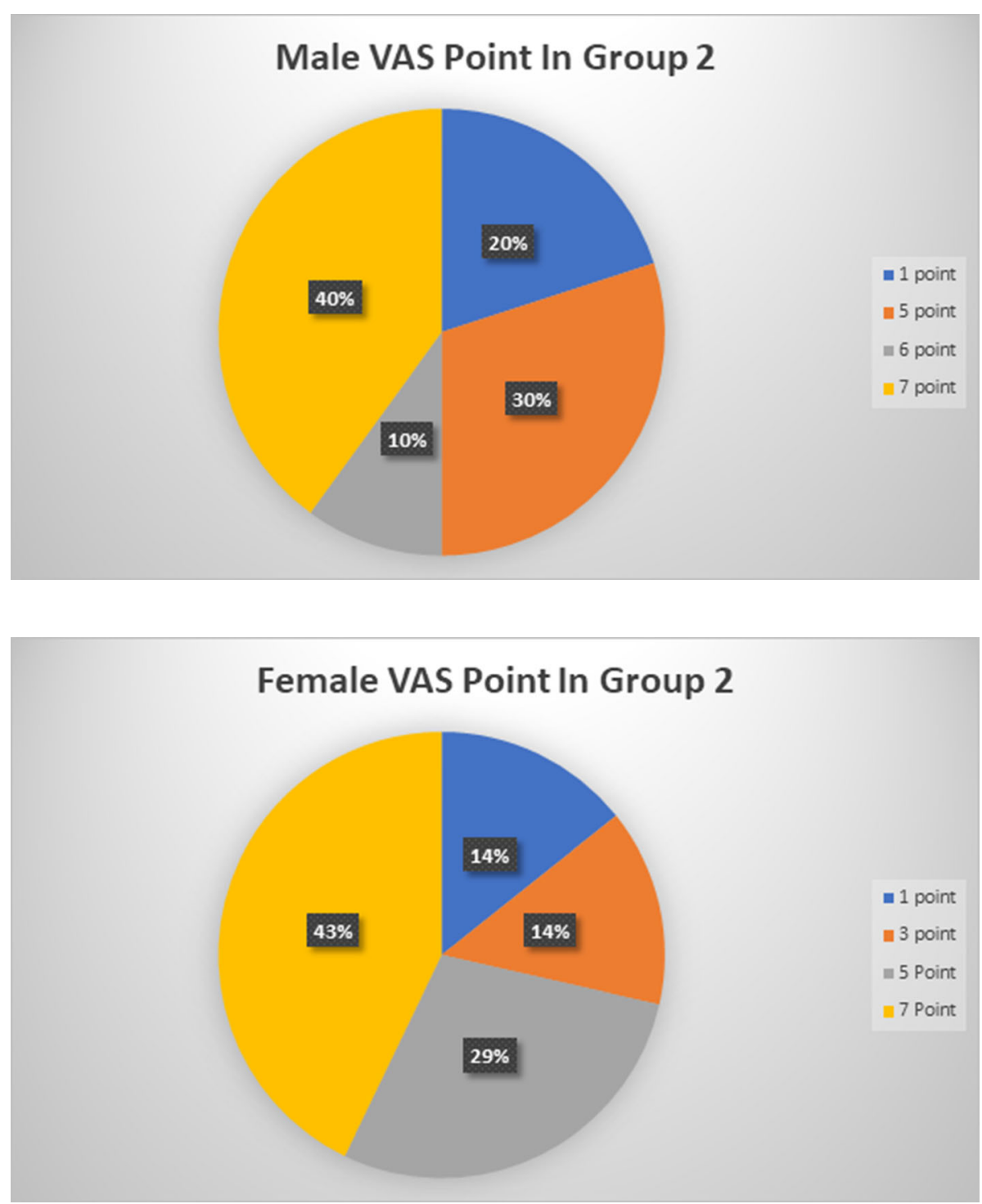

The mean value of PLR of all patients was 293.9 and the values ranged from 21.9 to 976.6. The mean value of PLR in men was $265.35 \pm 198.23$ whereas that of women was $348.98 \pm 236.22$. The mean value of PLR in Group 1 was $329.10 \pm 223.13$ whereas it was $250.55 \pm 197.33$ in Group 2.

No statistically significant difference was found between groups 1 and 2 in terms of gender difference. $(p=0.171)$.

No statistically significant difference was found between male and female patients in terms of VAS smell score $(p=0.198)$. No statistically significant difference was found between male and female patients in terms of NEU, LYMP, NLR values ( $p$ values $=0.935,0.369$, and 0.726 , respectively). A statistically significant difference was found between male and female patients in terms of PLT value, which was higher in female patients $(p=0.001)$. PLR value was higher in female patients even though no statistically significant difference was found between male and female patients in terms of PLR value $(p=0.063)$.

A statistically significant difference was found between Group 1 and Group 2 in terms of VAS smell score, NEU, LYMP, NLR. VAS smell score, NEU count, NLR value were found to be lower in Group 2 and LYMPH count was found to be higher. PLR value was found to be higher in Group 1 (Table 1).

\section{Discussions}

It was reported in the literature that 59 of 60 patients with COVID-19 had a loss of smell in Moein's study. The data in this study are reported to be similar to the loss of smell clinical characteristics of COVID-19 patients reported in a metanalysis of 3600 patients and 43 studies. The basis for 
Table $1 P$ values between groups

\begin{tabular}{lllr}
\hline & Group 1 & Group 2 & $P$ values \\
\hline Smell VAS Points & $10,00 \pm 0,00$ & $5,06 \pm 2,20$ & $<0,001$ \\
NEU & $7366,67 \pm 3581,72$ & $5900,00 \pm 2852,37$ & 0,019 \\
LYMP & $852,38 \pm 371,51$ & $1617,65 \pm 2190,13$ & 0,007 \\
NLR & $10,49 \pm 6,54$ & $6,30 \pm 4,99$ & $<0,001$ \\
PLT & $218,523,81 \pm 77,057,34$ & $241,294,12 \pm 172,733,59$ & 0,350 \\
PLR & $329,10 \pm 223,13$ & $250,55 \pm 197,33$ & 0,052 \\
\hline
\end{tabular}

loss of smell due to SARS-CoV-2 is not exactly clear even though it is well known that viruses can damage olfactory neuroepithelium [5]. Acute viral upper respiratory viral infections that damage this epithelium are the main cause of chronic olfactory dysfunction in daily practice and it is known that many viruses pass into the brain by cellular and paracellular transport mechanisms through this olfactory neuroepithelium [10]. Coronavirus enters the human host by a cell surface receptor known as angiotensin-converting enzyme 2 (ACE2), which is expressed in the human respiratory tract epithelium, lung parenchyma, vascular endothelium, kidney cells, and small intestinal cells. The ACE2 receptor binds to S1 spike glycoprotein in the viral envelope. The virus then enters the host cell through endocytosis [11]. Studies have shown that influenza viruses, coronaviruses, and other upper respiratory viruses' loss of smell peak at different months in various seasons. However, the difference in the pandemic period is that almost everyone who comes into contact with the novel coronavirus has a significant loss of smell without severe nasal congestion or inflammation [12]. Mechanical obstruction due to mucosal hypertrophy during active viral infection was thought to cause anosmia, but normal acoustic rhinometry results after infection suggest that patients may have different pathophysiology resulting in permanent anosmia and permanent damage [13]. Detailed neurological symptoms of hospitalized COVID-19 patients were recorded, hyposmia was detected in $5 \%$ of patients, and decreased sense of taste was found in about threequarters of these patients [14]. Loss of smell was detected in $44.7 \%$ of patients out of our 114 patient series. However, the difference of our study is that all patients were COVID19 swab PCR positive and all of them had pneumonia consistent with COVID-19. Our theory, which needs support, is that patients with loss of olfactory function have less pneumonia, are generally not hospitalized, have milder disease, and may be carriers in the disease. This is probably due to the immune mechanisms that occur in the olfactory region. It is possible that these mechanisms increase the loss of smell and protect the body from pneumonia. There is a need for studies to focus on these mechanisms and to point out that loss of smell may be a good indicator of prognosis in COVID-19 disease. Because none of our patients in Group 2 needed intensive care and advanced oxygen support.

The proportion of COVID-19 patients exhibiting olfactory disorders is not known. In general, most studies show that a significant number of people with loss of olfactory functions were not aware of their deficiencies until the test was performed and that both smell and taste abilities were weakly correlated with the results of smell and taste tests $[15,16]$. UPSIT test was used to evaluate olfactory function in COVID-19 patients in a study. Olfactory dysfunction was detected in 98 patients. Anemia was detected in $25 \%$, severe microsmia in $33 \%$, moderate microsmia in $27 \%$, mild microsmia in $13 \%$, and normosmia in $2 \%$ of the patients. We used VAS to evaluate the olfactory function in our study. The authorities' recommendation is methods with minimal risk of transmission during the challenging pandemic. 63 patients $(55.3 \%)$ had normosmia (VAS 10 points, Group 1) whereas 51 patients $(44.7 \%$, Group 2) had a loss of olfactory function. The VAS score in Group 2 varies between 1 and 7. 21 patients (18.4\%) gave 7 points, 15 patients (13.1\%) gave 5 points, 9 patients $(7.8 \%)$ gave 1 point, 3 patients $(2.6 \%)$ gave 6 points, and 3 patients (2.6\%) gave 3 points. A statistically significant difference was found between Group 1 and Group 2 in terms of VAS smell score. VAS smell score was found to be lower in Group 2.

Neutrophil lymphocyte (NLR) and platelet lymphocyte (PLR) ratios can be detected in peripheral complete blood count test. PLR value was found to be high in various peripheral vascular diseases, coronary artery diseases, some gynecological and hepatobiliary malignancies, and this was associated with poor prognosis. NLR increases in systemic inflammation, some gynecological and gastrointestinal cancers, and some cardiovascular diseases. Leukocyte and platelet values were found to be higher in patients with nasal polyps compared to healthy individuals in a study. This elevation in leukocyte value was found to 
be statistically significant $(p=0.001)$ [17]. NLR has been suggested to be used as a new marker showing systemic inflammation. NLR value has been shown to help determine short- and long-term mortality in acute coronary syndrome. Mortality also increases in patients with high NLR value. NLR was found to be higher in patients with sudden hearing loss compared to healthy individuals. In fact, non-response and poor prognosis were observed as the NLR value increased. Neutrophil and NLR values were found to be statistically significantly higher in patients with Bell Palsy compared to healthy individuals in another study [18-20]. Azab et al. showed that high PLR value increased mortality in their study on patients with non-ST-elevation myocardial infarction. It was emphasized that PLR value was more valuable than NLR value as an indicator of inflammation in patients with end-stage renal failure and PLR value was an important indicator of inflammation. Raungkaewmanee et al. reported that PLR value was an important prognostic factor in the patient's lifespan and that the lifespan of patients with PLR $>200$ was shorter than that of patients with PLR $<200$ [21-23]. PLR mean value was found to be higher than the control group, but the change was not statistically significant in a study conducted on patients with nasal polyps [17]. Chronic sinusitis patients were divided into two groups with and without nasal polyps and re-categorized according to recurrence status within these groups in a study. NLR and PLR values were found to be higher in the recurrent group in chronic sinusitis patients with nasal polyps $(p:<0.001,<0.001$, respectively). PLR value was found to be higher in the nonrecurrent group in chronic sinusitis patients without nasal polyps $(p=0.044)$ [24]. Leukocyte, NEU, NLR, and PLT values were found to be statistically significantly higher in patients with non-arteritic anterior ischemic optic neuropathy compared to the control group $(p:<0.001,<0.001,0.004$, and 0.037 , respectively). Monocyte and LYMP values were found to be higher even though it did not make a statistical difference in the patient group [25]. A statistically significant difference was found between Group 1 and Group 2 between NEU, LYMP, and NLR in our study. NEU count and NLR value were found to be lower in Group 2 and LYMP count was found to be higher. PLR value was found to be higher in Group 1 . There are studies in the literature emphasizing the importance of blood parameters related to the $2 \mathrm{nd}$, 7th, and 8th cranial nerves. In addition, various blood parameters studies have been conducted in pathological diseases with nasal inflammation. It is a fact that it causes cranial nerve disease and inflammation of the nasal mucosa. The count of LYMP and PLT were found to be high whereas the values of NEU, NLR, and PLR were low in patients with loss of smell. This is probably due to an unexplained inflammation pathway.
Blood parameters may be an important indicator of COVID-19-related olfactory nerve function loss. Neutrophil, lymphocyte, neutrophil/lymphocyte ratio, platelet, platelet/lymphocyte ratio, which are also used in nasal diseases, may be used to detect loss of smell, predict, and even determine the prognosis of loss of smell if supported by further studies.

Author Contributions All authors read and approved the final version of the manuscript.

Funding The authors received no financial support for the research and/or authorship of this article.

\section{Declarations}

Conflict of interest The authors declared no conflicts of interest with respect to the authorship and/or publication of this article.

Informed Consent Written and signed consent forms were obtained from all patients.

\section{References}

1. Eliezer M, Hautefort C, Hamel AL, Verillaud B, Herman P, Houdart E et al (2020) Sudden and complete olfactory loss function as a possible symptom of COVID-19. JAMA Otolaryngol Head Neck Surg 146(7):674-675. https://doi.org/1 0.1001/jamaoto.2020.0832

2. Luers JC, Klussmann JP, Guntinas-Lichius O (2020) The Covid19 pandemic and otolaryngology: what it comes down to? Laryngorhinootologie 99(5):287-291. https://doi.org/10.1055/a-1095-2344 (Epub 2020 Mar 26)

3. Giacomelli A, Pezzati L, Conti F, Bernacchia D, Siano M, Oreni $\mathrm{L}$ et al (2020) Self-reported olfactory and taste disorders in patients with severe acute respiratory coronavirus 2 infection: a cross-sectional study. Clin Infect Dis 71(15):889-890. https://doi.org/10.1093/cid/ciaa330

4. Yan CH, Faraji F, Prajapati DP, Boone CE, DeConde AS (2020) Association of chemosensory dysfunction and Covid-19 in patients presenting with influenza-like symptoms. Int Forum Allergy Rhinol 10(7):806-813. https://doi.org/10.1002/alr.22579 (Epub 2020 Jun 1)

5. Moein ST, Hashemian SM, Mansourafshar B, Khorram-Tousi A, Tabarsi P, Doty RL (2020) Smell dysfunction: a biomarker for COVID-19. Int Forum Allergy Rhinol 10(8):944-950. https://doi.org/10.1002/alr.22587 (Epub 2020 Jun 18)

6. Rockx B, Kuiken T, Herfst S, Bestebroer T, Lamers MM, Munnink BBO et al (2020) Comparative pathogenesis of COVID-19, MERS, and SARS in a nonhuman primate model. Science 368(6494):1012-1015. https://doi.org/10.1126/science .abb7314 (Epub 2020 Apr 17)

7. Lee Y, Min P, Lee S, Kim SW (2020) Prevalence and duration of acute loss of smell or taste in COVID-19 patients. J Korean Med Sci 35(18):e174. https://doi.org/10.3346/jkms.2020.35.e174

8. Mullol J, Mariño-Sánchez F, Valls M, Alobid I, Marin C (2020) The sense of smell in chronic rhinosinusitis. J Allergy Clin Immunol 145(3):773-776. https://doi.org/10.1016/j.jaci.2020 .01 .024

9. Mariño-Sánchez F, Santamaría A, de los Santos G, Alobid I, Mullol J (2020) Psychophysical olfactory testing in COVID-19: 
is smell function really impaired in nearly all patients? Int Forum Allergy Rhinol 10(8):951-952. https://doi.org/10.1002/alr.22639 (Epub 2020 Jun 25)

10. Doty RL (2008) The olfactory vector hypothesis of neurodegenerative disease: is it viable? Ann Neurol 63(1):7-15. https://doi.org/10.1002/ana.21327

11. Li YC, Bai WZ, Hashikawa T (2020) The neuroinvasive potential of SARS-CoV2 may play a role in the respiratory failure of COVID-19 patients. J Med Virol 92(6):552-555

12. Potter MR, Chen JH, Lobban NS, Doty RL (2020) Olfactory dysfunction from acute upper respiratory infections: Relationship to season of onset. Int Forum Allergy Rhinol 10(6):706-712. https://doi.org/10.1002/alr.22551 (Epub 2020 Apr 13)

13. Suzuki M, Saito K, Min W (2007) Identification of viruses in patients with postviral olfactory dysfunction. Laryngoscope 117:272-277

14. Mao L, Jin H, Wang M, Hu Y, Chen S, He Q et al (2020) Neurologic manifestations of hospitalized patients with coronavirus disease 2019 in Wuhan China. JAMA Neurol 77(6):683-690. https://doi.org/10.1001/jamaneurol.2020.1127

15. Wehling E, Nordin S, Espeseth T, Reinvang I, Lundervold AJ (2011) Unawareness of olfactory dysfunction and its association with cognitive functioning in middle aged and old adults. Arch Clin Neuropsychol 26(3):260-269

16. Soter A, Kim J, Jackman A, Tourbier I, Kaul A, Doty RL (2008) Accuracy of self-report in detecting taste dysfunction. Laryngoscope 118(4):611-617

17. Atan D, Ozcan KM, Koseoglu S, Ikinciogullari A, Cetin MA, Ensari S et al (2015) New predictive parameters of nasal polyposis: neutrophil to lymphocyte ratio and platelet to lymphocyte ratio. Kulak Burun Bogaz Ihtis Derg 25(2):97-101. https://doi.org/10.5606/kbbihtisas.2015.01947

18. Bhat T, Teli S, Rijal J, Bhat H, Raza M, Khoueiry G et al (2013) Neutrophil to lymphocyte ratio and cardiovascular diseases: a review. Expert Rev Cardiovasc Ther 11:55-59
19. Ulu S, Ulu MS, Bucak A, Ahsen A, Yucedag F, Aycicek A (2013) Neutrophil-to-lymphocyte ratio as a new, quick, and reliable indicator for predicting diagnosis and prognosis of idiopathic sudden sensorineural hearing loss. Otol Neurotol 34:1400-1404

20. Bucak A, Ulu S, Oruc S, Tekin MS, Karakaya F, Aycicek A (2014) Neutrophil-to-lymphocyte ratio as a novelpotential marker for predicting prognosis of Bell palsy. Laryngoscope 124:1678-1681

21. Azab B, Shah N, Akerman M, McGinn JT Jr (2012) Value of platelet/lymphocyte ratio as a predictor of allcause mortality after non-ST-elevation myocardial infarction. J Thromb Thrombolysis 34:326-334

22. Turkmen K (2013) Platelet-to -lymphocyte ratio: one of the novel and valuable platelet indices in hemodialysispatients. Hemodial Int 17:670

23. Raungkaewmanee S, Tangjitgamol S, Manusirivithaya S, Srijaipracharoen S, Thavaramara T (2012) Platelet to lymphocyte ratio as a prognosticfactor for epithelial ovarian cancer. J Gynecol Oncol 23:265-273

24. Boztepe OF, Gün T, Demir M, Gür OE, Ozel D, Dogru H (2016) A novel predictive marker for the recurrence of nasal polyposis following endoscopic sinus surgery. Eur Arch Otorhinolaryngol 273(6):1439-1444. https://doi.org/10.1007/s00405-015-3753-Z (Epub 2015 Aug 19)

25. Polat O, Yavas GF, Inan S, Inan UU (2015) Neutrophil-to-lymphocyte ratio as a marker in patients with non-arteritic anterior ischemic optic neuropathy. Balkan Med J 32(4):382-387. https://doi.org/10.5152/balkanmedj.2015.15689 (Epub 2015 Oct 1)

Publisher's Note Springer Nature remains neutral with regard to jurisdictional claims in published maps and institutional affiliations. 